\title{
On Application of PHY Layer Abstraction Techniques for System Level Simulation and Adaptive Modulation in IEEE 802.11ac/ax Systems
}

\author{
Roger Pierre Fabris Hoefel and Oscar Bejarano
}

\begin{abstract}
This paper investigates the application in IEEE 802.11ac/ax systems of the received bit information rate (RBIR) technique in order to estimate the effective signal-tointerference-plus-noise ratio used to abstract the physical layer (PHY) performance in system level simulations. The RBIR technique is evaluated based on simulation results of IEEE 802.11ac PHY operating over canonical flat fading and spatial-correlated frequency selective single-user and multiuser multiple input multiple output channels. We have concluded that the RBIR PHY abstraction methodology is accurate enough to provide first order insights on system level performance and design options with reduced computational complexity. Finally, the application of RBIR PHY abstraction scheme to schedule the modulation and code schemes on the flight to achieve a target quality-ofservice is also described.
\end{abstract}

Index Terms-802.11ac, 802.11ax, MIMO, OFDM, RBIR, modulation and code schemes, scheduling.

\section{INTRODUCTION}

The main target of the 2013 IEEE 802.11ac amendment [1] is to improve the physical layer (PHY) throughput (or link level throughput) of the 2009 IEEE 802.11n amendment [2], i.e., the maximum theoretical throughput is increased from $600 \mathrm{Mbps}$ to $6.77 \mathrm{Gbps}$ in wireless local area networks (WLANs) based on the IEEE 802.11ac specification. Basically, PHY data rate improvements in IEEE 802.11ac WLANs have been achieved by operating with bandwidths (BW) of 20/40/80/80+80 $/ 160 \mathrm{MHz}$; orthogonal frequency division multiplexing (OFDM) single-user (SU) multiple input

Roger Pierre Fabris Hoefel, Electrical Engineering Department, Federal University of Rio Grande do Sul, Porto Alegre, Rio Grande do Sul, Brazil, E-mail: roger.hoefel@ufrgs.br

Oscar Bejarano, Cisco Systems Inc., Cleveland, USA, E-mail: obejarano@alumni.rice.edu

The Associate Editors coordinating the review of this manuscript and approving it for publication were Profs. Cecílio Pimentel and Marcelo da Silva Pinho.

A preliminary version of this paper was presented in XXXIII Simpósio Brasileiro de Telecomunicações (SBrT’15), Juiz de Fora, MG, Brazil, September $1-4,2015[26]$

Digital Object Identifier: 10.14209/jcis.2016.18 multiple output (MIMO) with up to 8 layers; quadrature amplitude modulation (QAM) schemes with cardinalities that range from 2 to 256 [3]. The optional implementation of downlink multi-user MIMO (DL MU-MIMO) allows improving the system throughput when the channel is loaded with stations (STAs) that have a smaller number of antennas than the number antennas deployed at the access point (AP), i.e., a common scenario in corporative WLANs loaded with small form factor devices, such as smartphones and tablets [4].

The scarcity of "front-beach" spectrum below 6 $\mathrm{GHz}$ and the explosive demand of number of devices and traffic have driven intensive research and development (R\&D) activities to cope with these challenges upon restrictions of capital expenditure/ operational expenditure (CAPEX/OPEX). One medium term solution that has been considered in the scope of Third Generation Partnership Project (3GPP) is the development of Long Term Evolution Licensed Assisted Access (LTE-LLA) specification to operate at the unlicensed $5 \mathrm{GHz}$ band for traffic offloading [5]. A typical LTE-LLA configuration uses a primary carrier in licensed spectrum (e.g., $1.8 \mathrm{GHz}$ ) for control and data planes, and a secondary carrier in the unlicensed spectrum (e.g., $5 \mathrm{GHz}$ ) for data offload.

In the scope of WLANs community, the Task Group (TG) 802.11ax was created in March 2014 [6] to develop a new IEEE 802.11 amendment to face with the following challenges: (i) exponential increase of traffic and number of devices in dense networks; (ii) competition of LTE-LLA; (iii) demands of telecom operators to offload traffic; (iv) necessity of device vendors to offer better user experience urged in corporative and consumer electronics market segments; $(v)$ pressure of chip set 
vendors to create a WiFi market after the IEEE $802.11 \mathrm{ac}$ amendment. The Draft 1.0 of IEEE 802.11ax is expected to be approved in 2016, while the Final Spec is forecasted to 2019. The IEEE 802.11ax is also labeled as High Efficiency WLANs (HEW) in TG 802.11ax documents.

The IEEE 802.11ax amendment aims at increasing per area throughput efficiency in dense networks deployed in both outdoor and indoor environments with major concerns about power consumption instead of focusing per-link throughput in indoor networks, as focused on the earlier IEEE 802.11 specifications. A non-extensive list of candidate technologies include downlink and uplink MUMIMO, orthogonal frequency division multiple access (OFDMA), massive MIMO, MIMO precoding, long cyclic prefix (CP), fractional frequency reuse, full-duplex. There is also a strong trend that the medium access control (MAC) protocol can be based on the Carrier Sense Multiple Access (CSMA) algorithm due to the distributed nature of WLANs operating in different administrative domains. However, the introduction of scheduling to handle with the dramatic increase of the number of devices as well as the uncontested demand of qualityof-service (QoS) by consumers and corporations has been a topic of intensive research in TGax.

The application of cross-layer techniques to design MAC and PHY protocols has driven the TGax 802.11ax to define a uniform set of PHY abstraction techniques in order to evaluate the performance of the different specification proposals considered in TG 802.11ax [7]. In Section II, we contextualize the contributions of this paper to recent $R \& D$ activities that have been carried out in IEEE TG 802.11ax on the application of quality metric (QM) models for enabling system level simulations (SLS) with affordable computational complexity. The remaining sections of this paper have the following layout: Section III describes the received bit information rate (RBIR) technique used to calculate the effective signal-to-interference-plus-noise ratio (ESINR) used to estimate the packet error rate (PER) without extensive PHY simulations for each channel realization. Section IV presents simulation results to validate the RBIR PHY abstraction technique in the framework of IEEE 802.11ac/x WLANs over a set of realistic operational setups. Section V proposes the application of PHY abstraction techniques to radio resource control (RRC), i.e., the RBIR metric is used to schedule in real time the modulation and code scheme (MCS) that satisfies the target QoS. Section VI presents our final conclusions.

Finally, we observe that the IEEE 802.11ax PHY is not defined yet. Therefore, we follow the same approach that has been used in the TGax, i.e., the simulation results are based on the IEEE 802.11ac amendment [7]. Notice that analyzes of PHY layer abstraction techniques is also relevant for next generation of IEEE 802.11ac chipsets since the WiFi semiconductor industry has been developing $R \& D$ activities to improve the performance of IEEE 802.11 ac second wave products (e.g. DL MU-MIMO techniques; BW of 80 and $160 \mathrm{MHz}$ ) in order to achieve a maximum PHY throughput of $\sim 3.5 \mathrm{Gbps}$.

\section{RELATED WORKS AND MAIN CONTRIBUTIONS}

Research on application of PHY abstraction techniques for SLS was primarily carried out at the beginning of broadband cellular networks standardization process in 3GPP [8], 3GPP2 [9] and IEEE 802.16 Working Group (WG) [10]. Application of PHY abstraction techniques in LTE system level simulators can be found in [11] and [12] in the context of Brazilian and international, respectively, research community.

The process of defining a methodology to PHY abstraction in IEEE 802.11 TGs [13-14] also occurred concomitantly with the activities developed at 3GPP and IEEE 802.16 WG [8-10]. However, WiFi semiconductor players only have demanded strong attention upon this topic at the beginning of IEEE 802.11ax standardization process [7] due to the paradigm change from link level design to cross-layer optimization of PHY and MAC protocols that demand efficient design tools in order to evaluate the system performance over typical usage cases forecasted in ultra-dense network deployments [4]. Several PHY layer techniques have been investigated in TG 802.11ax, e.g., Exponential Mapping; Mutual 
Information assuming Bit-Interleaved Code Modulation (BICM); Mutual Information per Bit and RBIR [14]. The technique of mapping the SINR observed at the OFDM MIMO detector output to an effective SINR (ESINR) scalar metric based on mutual information (MI) [15] has been chosen by the TG 802.11ax to implement PHY abstraction algorithms to evaluate PHY and MAC protocols during the IEEE 802.11ax standardization process [16].

Public studies on this subject have been mainly restricted to TGax meetings. Reference [17] is one of few peer-to-peer review papers where the application of PHY abstraction techniques in WLANs is investigated. However, this paper only shows simulation results based on the IEEE 802.11n amendment, assuming 2x2 SU-MIMO spatial multiplexing with ideal channel state information (CSI) at receiver side (RX).

In this paper, we have investigated the application of RBIR for ESINR mapping in IEEE 802.11ax systems assuming realistic synchronization and channel estimation schemes over settings not widely analyzed in TGax documents in the conformation of PHY abstraction, such as, the effects of the number of SU-MIMO spatial streams (SS) and DL MU-MIMO with block diagonalization (BD) pre-coding over spatial correlated and frequency selective TGn SUMIMO and TGac MU-MIMO channel models.

Fig. 1 shows a simplified block diagram for the transmission of MU data field physical layer protocol dada unit (PPDU) using the $20 \mathrm{MHz}, 40 \mathrm{MHz}$ or 80 $\mathrm{MHz}$ BW [1]. Notice that multiple users are processed independently before the spatial mapping block (i.e., the precoding scheme) in the IEEE 802.11ac PHY. A detailed description of the IEEE 802.11ac PHY layer can be found in [3]. A concise description of main operational characteristics of IEEE 802.11ac PHY is presented in [18].

The validation of the IEEE 802.11 ac simulator used in this paper, whose main characteristics are shown in Tab. I, is developed in [19].

In [20] we describe three different channel estimation schemes suitable to operate in IEEE 802.11n devices: Least Squares (LS); Time Delay
Truncation (TDT) and Model-Based (MB). Founded on analytical and simulation results of channel estimation mean square error (MSE) and MAC protocol data unit (MPDU) PER, we have concluded that the TDT presents a superior performance in low

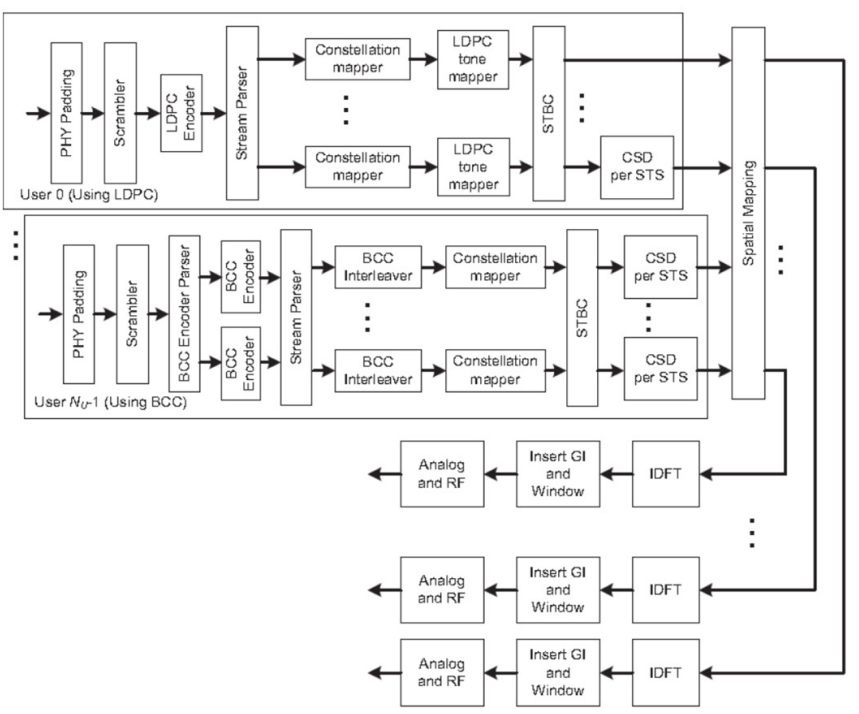

Fig. 1. "Transmitter block diagram for the Data Field MU PPDU" [1]. Acronyms: BCC (Binary Convolutional Code); LDPC (Low Density Paritt Check); STBC (Spatial-Time Block Coding); CSD (Cyclic Shift-Diversity); IDFT (Inverse Discrete Fourier Transform); GI (Guard Interval); RF (Radio Frequency).

Table I. Basic parameters of IEEE 802.11 ac simulator [19].

\begin{tabular}{c|c|c|c}
\hline Parameter & Value & Parameter & Value \\
\hline $\begin{array}{c}\text { Carrier } \\
\text { Frequency }\end{array}$ & $5.25 \mathrm{GHz}$ & MCS & $0-9$ \\
\hline Bandwidth & $\begin{array}{c}20 \mathrm{MHz}, 40 \mathrm{MHz}, \\
80 \mathrm{MHz}\end{array}$ & $\begin{array}{c}\text { Number of } \\
\text { Spatial Streams }\end{array}$ & 1 to 8 \\
\hline GI Length & $800 \mathrm{~ns}$ & Synchronization & $\begin{array}{c}\text { Auto- } \\
\text { Correlation }\end{array}$ \\
\hline Modulation & BPSK, QPSK, & MIMO Channel & Least Squares \\
& $16-\mathrm{QAM}$, & Estimation & \\
\hline Binary & Code rate: & Channel & $\begin{array}{c}\text { Hard and } \\
\text { Code (BCC) }\end{array}$ \\
& $\mathrm{r}=1 / 2, \mathrm{r}=2 / 3$, & Decoder & $\begin{array}{c}\text { Soft-Decision } \\
\text { Viterbi } \\
\text { Decoding }\end{array}$ \\
\hline
\end{tabular}

SNR regime, while the LS channel estimation schemes outperforms dramatically the other two schemes in the medium and high SNR regions. Consequently, we have chosen the LS channel estimation scheme based on the results shown in [20] as well as its low computational burden. However, we recognize that the design of channel estimation schemes to maximize throughput is still an open R\&D topic in the WiFI community. 
Finally, we must reemphasize that the results shown in this paper assume realistic LS channel estimation scheme [2, pp. 98], and time and frequency synchronization over spatial and frequency selective TGn/ac MIMO channel models. This is one of the main differential aspects of this paper in relation to open technical reports and public presentations done in TGax meetings [7, 13-14], where both perfect synchronization and CSI are assumed.

\section{RBIR IN THE CONTEXT OF IEEE 802.11 AC/AX}

\section{A. Effective SINR}

The performance of OFDM MIMO systems depends on the statistics of the SINR measured at the MIMO detector output (i.e., the post-processing SINR). The application of quality metric (QM) techniques to evaluate the OFDM MIMO system performance with small complexity is founded on the mapping the set of post-processing SINRs $\quad\left\{S_{N} R_{n, k}, n=\right.$ $\left.1, \cdots, N_{s S}, k=1, \cdots N_{s c}\right\}$ for all $N_{s c}$ data subcarriers (SC) for each one of the $N_{s s}$ spatial streams (SS) to an ESINR. This ESINR allows that the PER estimated over an OFDM MIMO additive white Gaussian noise (AWGN) channel be equivalent to that one estimated with full PHY simulation over the block fading channel realization where the ESINR has been determined [15].

The general definition of the effective SINR mapping (ESM) for OFDM MIMO systems is given by [7]

$$
\operatorname{SINR}_{e f f}=\alpha \Phi^{-1}\left(\frac{1}{N_{s s}} \frac{1}{N_{s c}} \sum_{n=1}^{N_{S S}} \sum_{k=1}^{N_{S c}} \Phi\left(\frac{\operatorname{SIN} R_{n, k}}{\beta}\right)\right),
$$

where $\operatorname{SINR}_{n, k}$ denotes the SINR at MIMO detector output at the $k$ th SC of the $n$th SS, $\Phi($.$) is the ESM$ function. The parameters $\alpha$ and $\beta$ are tuning factors, which should be obtained numerically to minimize the MSE between the PER obtained with full PHY layer simulation and the PER estimated using the PHY layer abstraction technique. In this paper, we assume $\alpha=\beta=1$. Therefore, only the lower bound of RBIR PHY abstraction technique application in IEEE 802.11ac/x WLANS is evaluated in this paper since no further optimization is carried out. Notice that the same approach also has been used in some contributions presented in the TGax meetings [14].
Simulation studies on the effects of tuning factors on the accuracy of RBIR PHY abstraction methodology can be found in [7,21].

The PHY abstraction concept to estimate the PER is depicted in Fig. 2. The objective is to determine the PER for each transmitted MPDU with low computational burden. To accomplish it, the vector composed of the set of $\left\{\operatorname{SINR}_{n, k}, n=1, \cdots, N_{s S}, k=\right.$ $\left.1, \cdots N_{s c}\right\}$ estimated at the output of MIMO detector is mapped to a scalar ESINR using (1). Then, the ESINR is used to get the PER using a reference curve obtained with full PHY layer simulation over AWGN MIMO channels. One important criterion for the evaluation of PHY abstraction algorithms for SLS is the matching between the PER ${ }_{1}$ obtained using link level simulation (LLS) with the $\mathrm{PER}_{2}$ estimated using PHY abstraction techniques.

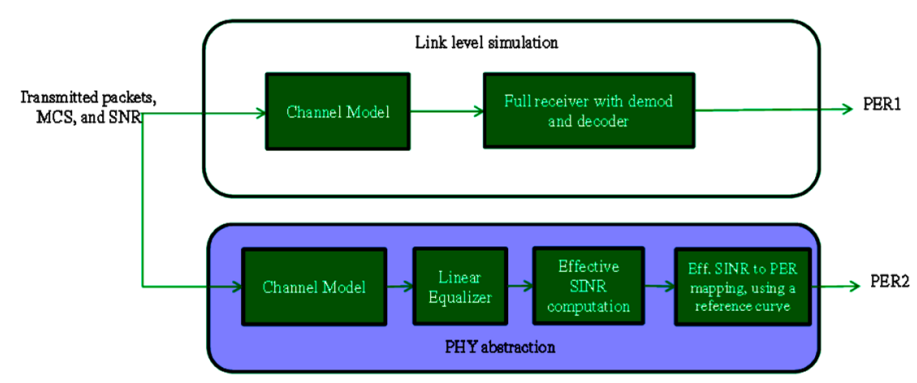

Fig. 2. Block diagram to visualize the PHY abstraction concept [22].

Assuming an M-ary modulation with cardinality $\mathrm{M}$, the received bit information rate (RBIR) metric uses an ESM function given by [15]

$$
\Phi(\gamma)=E_{X Y}\left\{\log _{2} \frac{f(y / X, \gamma)}{\sum_{m=1}^{M} P(X) \cdot f(y / X, \gamma)}\right\},
$$

where $X$ is a complex discrete random variable (r.v.) with probability mass function $P(X)$ that models the transmit symbols from the $\mathrm{M}$-ary constellation, $Y$ is a continuous r.v. that models the channel output with SINR equal to $\gamma$, and $f(y / X, \gamma)$ denotes the conditional probability density function (pdf) of the r.v. Y measured at the AWGN channel output given the r.v. $\mathrm{X}$ and $\gamma$.

Eq. (2) can be expanded to a numerical expression as follows:

$$
\begin{aligned}
& \Phi\left(\gamma_{\mathrm{n}, \mathrm{k}}\right)=\log _{2} M- \\
& \frac{1}{M} \sum_{i=1}^{M} E_{U}\left\{\log _{2}\left(\frac{\sum_{j=1}^{M} \exp \left[-\left|\sqrt{\gamma_{\mathrm{n}, \mathrm{k}}}\left(s_{j}-s_{i}\right)+U\right|^{2}\right]}{\exp \left[-|U|^{2}\right]}\right)\right\},
\end{aligned}
$$


where $\mathrm{E}_{\mathrm{U}}$ denotes the expected value of the r.v. $U, \gamma_{\mathrm{n}, \mathrm{k}}$ denotes the SINR measured at the $k$ th SC in the $n$th SS, $s_{k}$ and $s_{m}$ are constellation points with normalized energy and $U$ is a circular symmetric complex Gaussian (CSCG) r.v. with zero mean and unitary variance [10].

Finally, we point out that according with (1), different channel realizations with the same SINR $R_{\text {eff }}$ have the same PER for a given coding block length. Therefore, we can use the PER estimated over the AWGN channel as a reference curve to implement the mapping from the estimated SINR $R_{\text {eff }}$ to PER over block fading channels.

\section{B. MIMO AWGN CHANNEL}

The MIMO AWGN channel model was used as comparison criterion for the PHY performance evaluation of the proposals submitted during the IEEE 802.11n standardization process [3, pp. 122]. The MIMO matrix $H$ (cf. Eq. 4a) is obtained taking the first $N_{r x}$ by $N_{t x}$ elements from the Fourier matrix with dimension $N=\max \left(N_{r x}, N_{t x}\right)$, where $W=$ $\exp (-j 2 \pi / N) . N_{t x}$ and $N_{r x}$ denote the number of transmit and receive antennas, respectively. Notice that the rows and columns of the Fourier matrix are orthogonal. Therefore, the implementation of a decorrelator receiver allows decoupling the transmitted streams without noise enhancement when ideal CSI is assumed.

$$
\mathbf{H}=\left[\begin{array}{ccccc}
1 & 1 & 1 & \cdots & 1 \\
1 & W^{1} & W^{2} & \cdots & W^{(N-1)} \\
1 & W^{2} & W^{4} & \cdots & W^{2(N-1)} \\
\vdots & \vdots & \vdots & \ddots & \vdots \\
1 & W^{(N-1)} & W^{(N-1) 2} & \cdots & W^{(N-1)^{2}}
\end{array}\right] .
$$

The output of the OFDM MIMO channel can be modeled by the column vector $\mathbf{r}_{k}[\mathrm{~m}] \in \mathbb{C}^{\mathrm{N}_{\mathrm{rx}}}$ :

$$
\mathbf{r}_{k}[\mathrm{~m}]=\mathbf{H}_{\boldsymbol{k}}[\mathrm{m}] \mathbf{x}_{\boldsymbol{k}}[\mathrm{m}]+\mathbf{n}_{\boldsymbol{k}}[\mathrm{m}]
$$

where the column vector $\boldsymbol{x}_{\mathrm{k}}[\mathrm{m}] \in \mathbb{C}^{\mathrm{N}_{\mathrm{tx}}}$ denotes the $m$ th transmitted OFDM symbol at $k$ th subcarrier, and $\mathbf{H}_{\mathbf{k}}[\mathrm{m}] \in \mathbb{C}^{\mathrm{N}_{\mathrm{rx}} \times \mathrm{N}_{\mathrm{tx}}}$ is the channel matrix observed at the receiver input for the $m$ th transmitted OFDM symbol at $k$ th subcarrier. The additive noise is circular symmetric Gaussian r.v. with variance $N_{0}$, $\mathbf{n} \sim \mathrm{CN}\left(0, N_{o} \mathbf{I}_{\mathrm{N}_{\mathrm{rx}}}\right)$, where $\mathbf{I}_{\mathrm{N}_{\mathrm{rx}}}$ is a square identity matrix with size $N_{r x}$. Note that for the AWGN channel the MIMO channel matrix is the same for all subcarriers.

\section{RECEIVED BIT INFORMATION RATE OVER MIMO AWGN CHANNEL}

Fig. 3a shows the RBIR as a function of the SNR for the $4 x 4$ MIMO AWGN channel assuming LS channel estimation scheme and minimum mean squared error (MMSE) receiver. Assuming a SNR of $10 \mathrm{~dB}$, the RBIR is 1.0 and 1.97 for binary phase-shift keying (BPSK) and quaternary phase-shift keying (QPSK) modulation schemes, respectively. Notice that the maximum RBIR values for BPSK and QPSK are 1 and 2, respectively. Assuming the same SNR of 10 $\mathrm{dB}$, the RBIR is only $70 \%, 48 \%$ and $37 \%$ of its maximum value of 4 (16-QAM), 6 (64-QAM) and 8 (256-QAM), respectively.

Fig. 3b shows that the effects of non-ideal channel estimation decreases the RBIR directly proportional to the modulation cardinality. For instance, when perfect CSI is assumed, a RBIR of 5 bits is obtained with a SNR of $17 \mathrm{~dB}$ and $15 \mathrm{~dB}$ for 64-QAM and 256-QAM, respectively. However, SNR of $20 \mathrm{~dB}$ (64-QAM) and $18.5 \mathrm{~dB}$ (256-QAM) are demanded when the realistic LS channel estimation scheme is assumed. Therefore, we can infer that performance losses that range from 3 to $3.5 \mathrm{~dB}$ can occur due to imperfect CSI over MIMO AWGN channels with MMSE receivers and LS channel estimation scheme.

Simulation results (see results summarized in Tab. II) have shown that the PER in the medium SNR regime presents a small dependence with the $\mathrm{BW}$ and number of antennas even when we assume MIMO AWGN channels with realistic synchronization, LS channel estimation scheme, and MMSE MIMO detectors. The Fourier matrix that models the AWGN channel (see. 4a) is unitary if scaled correctly. Therefore, the symbols at the output of MIMO detector channel have a small intra-spatial stream interference with an MMSE linear filter even when realistic synchronization and channel estimation schemes are assumed. Finally, observe that the results shown in Fig. 3 can also be used to estimate the first order performance evaluation for other AWGN MIMO channel configurations (e.g., different number of antennas) due to the aforementioned remarks. 

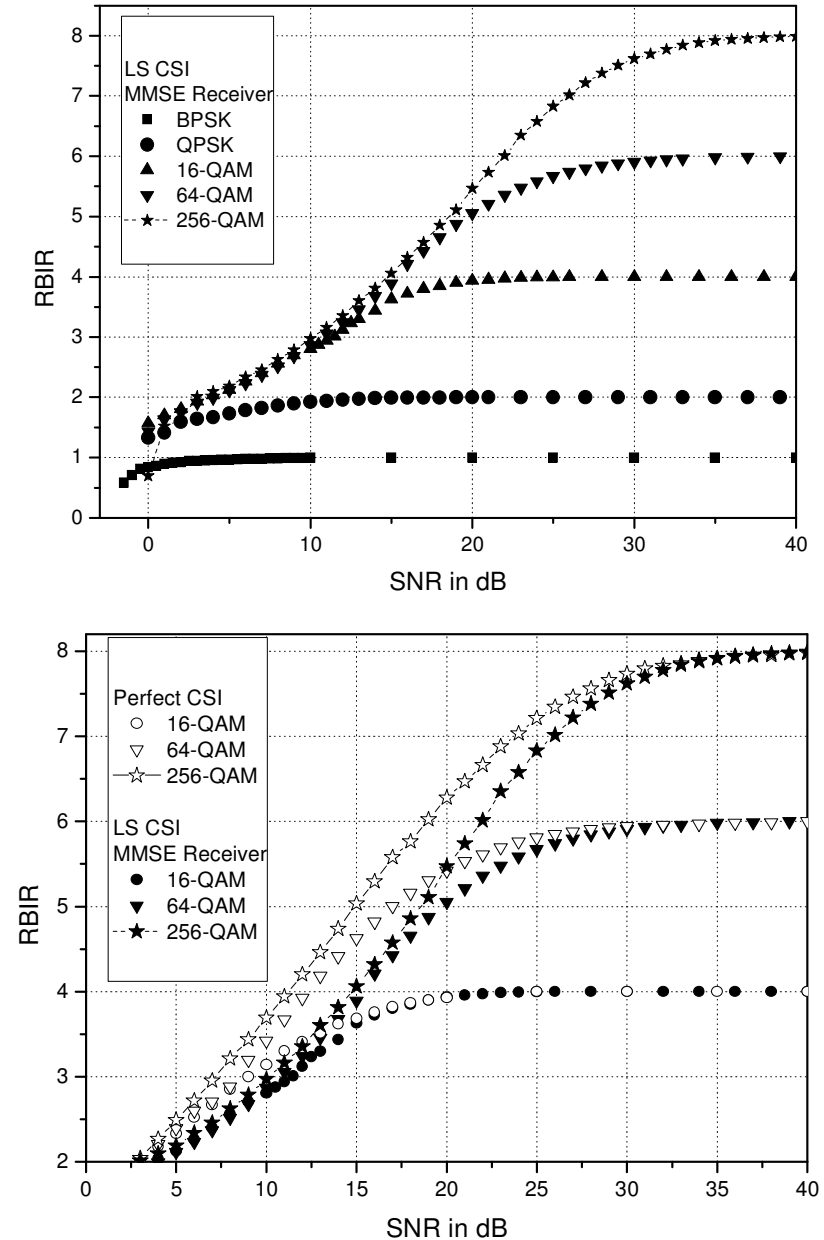

Fig. 3. RBIR as function of SNR over AWGN channel: (a) Perfect CSI; (b) Perfect and LS CSI.

Table II - SNR in $\mathrm{dB}$ to obtain a packet error rate of $1 \%$ as function of bandwidth and AWGN MIMO channel dimensionality for MCS4 (16-QAM, BCC with $\mathrm{r}=3 / 4)$, MCS6 (64-QAM, BCC with $\mathrm{r}=3 / 4$ ) and MCS8 (256-QAM, BCC with $\mathrm{r}=3 / 4)$. Packet size of 1500 bytes. Soft-decision Viterbi decoding.

\begin{tabular}{c|c|c|c|c|c|c}
\hline & \multicolumn{3}{|c|}{ BW=20 MHz } & \multicolumn{3}{c}{ BW=80 MHz } \\
\hline & $1 \times 1$ & $2 \times 2$ & $8 \times 8$ & $1 \times 1$ & $2 \times 2$ & $8 \times 8$ \\
\hline MCS4 & $16.2 \mathrm{~dB}$ & $16.7 \mathrm{~dB}$ & $16.5 \mathrm{~dB}$ & 16.0 & 16.6 & 16.6 \\
\hline MCS6 & $22.1 \mathrm{~dB}$ & $22.4 \mathrm{~dB}$ & $22.2 \mathrm{~dB}$ & 21.9 & 22.3 & 22.1 \\
\hline MCS8 & $27.2 \mathrm{~dB}$ & $27.8 \mathrm{~dB}$ & $27.6 \mathrm{~dB}$ & 27.3 & 27.8 & 27.6 \\
\hline
\end{tabular}

\section{PER over AWGN Using PHY Layer Simulation}

Tab. III, assuming the AWGN 2x2 MIMO channel, shows that our IEEE 802.11ac PHY simulation results present a close agreement with the PHY simulation results presented in [7], i.e., there is a first order consistence of the results shown in this paper. These results assume perfect CSI and decorrelator receiver, i.e., the same assumptions used in [7].
Table III - SNR in dB to obtain a PER of $1 \%$. AWGN $2 \times 2$. Packet sizes of 250/500/1000/1000 bytes for MCS1, MCS4, MCS6 and MCS8 (256-QAM) Soft-decision Viterbi decoding.

\begin{tabular}{c|c|c|c|c}
\hline & MCS1 & MCS4 & MCS6 & MCS8 \\
\hline $\begin{array}{c}\text { Simulation } \\
\text { Perfect CSI }\end{array}$ & $3.5 \mathrm{~dB}$ & $12.8 \mathrm{~dB}$ & $18.8 \mathrm{~dB}$ & $24.3 \mathrm{~dB}$ \\
\hline $\begin{array}{c}\text { Ref. [7] } \\
\text { Perfect CSI }\end{array}$ & $4.0 \mathrm{~dB}$ & $13.0 \mathrm{~dB}$ & $18.5 \mathrm{~dB}$ & $24.5 \mathrm{~dB}$ \\
\hline
\end{tabular}

Fig. 4 shows the MPDU PER as a function of SINR over a MIMO AWGN channel with BW of $20 \mathrm{MHz}$. The receiver implements LS channel estimation and MMSE receiver with soft-decision Viterbi decoding. An MPDU payload of 1500 bytes is used. Finally, notice that for the AWGN channel, the ESNR and SNR have the same logical meaning, as we have explained in the previous subsection.

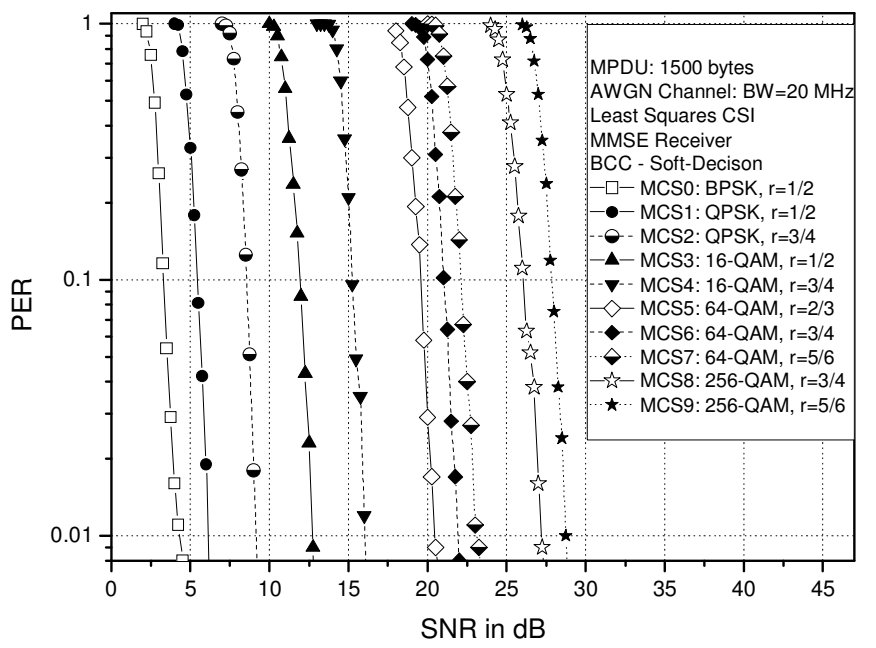

Fig. 4. PER vs. SNR in $\mathrm{dB}$ for $\mathrm{LS}$ channel estimation.

Fig. 5 shows that a realistic receiver with LS channel and MMSE MIMO detector presents performance losses between 2.5 (MCS2) and $3 \mathrm{~dB}$ (MCS8) in relation to an hypothetical system with perfect CSI and ideal decorrelator receiver, i.e., these results are consistent with the losses in the RBIR due to imperfect CSI at RX side shown in Fig. $3 b$.

\section{E. PER Using PHY Layer Abstraction}

The algorithm based on RBIR and ESINR to estimate the PER in OFDM MIMO systems is described as follows: 


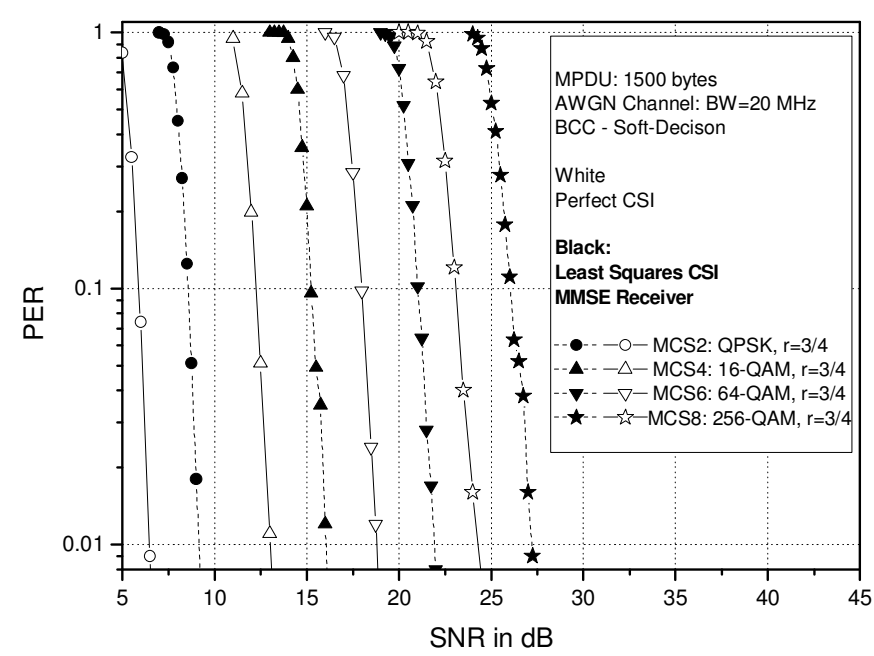

Fig. 5. Effects of imperfect channel estimation on the PER.

Step 1. Determine the $\operatorname{SINR}_{n, k}$ at the output of MIMO detector for all SSs $\left(n=1, \ldots, N_{s s}\right)$ and SCs $\left(k=1, \ldots, N_{s c}\right)$. These values of SINR must be obtained for the MCS being evaluated in the IEEE 802.11ac/ax simulator that consistently abstract the details of PHY layer. Notice that the $S I N R_{n, k}$ depends on both the modulation scheme and code rate specified for each MCS.

Step 2. Use the mapping function specified in (3), with the appropriate modulation constellation and the SINR measured for all SSs and SCs $\left(\gamma_{\mathrm{n}, \mathrm{k}}\right)$, to get the set $\left\{\Phi\left(\gamma_{\mathrm{n}, \mathrm{k}}\right), n=1, \cdots, N_{s s}, k=1, \cdots N_{s c}\right\}$.

Step 3. Use (5) to calculate the RBIR using the set of SINRs specified at step (2).

$R B I R=\frac{1}{N_{s s}} \frac{1}{N_{S c}} \sum_{n=1}^{N_{S S}} \sum_{k=1}^{N_{S c}} \Phi\left(\operatorname{SINR}_{n, k}\right)$.

Step 4. A table look up (TUL) procedure is utilized to map the RBIR to the ESINR. In this paper, we use the data shown in Fig. 3. Notice that the LS channel estimation scheme and realistic synchronization are assumed, instead of the oversimplified ideal channel estimation and synchronization assumptions postulated in $[7,16]$.

Step 5. Use the ESINR calculated at step (4) to estimate the PER probability using a TUL procedure to perform the mapping from ESINR to PER. These tables are obtained from full PHY simulation over the AWGN channel (e.g., see Fig. 4).

In this paper, unless otherwise noticed, we use the results shown in Fig. 4, which depend upon MPDU length, channel estimation scheme and receiver type. Finally, we emphasize that this procedure can be used in a time-based (i.e. no snapshot-based) block fading channel since the effective SINR is estimated for each different channel realization. In this paper, we consider that the interference does not change during each MPDU transmission.

\section{EVALUATION OF RBIR IN IEEE 802.11 AC/AX WLANS}

\section{A. Histogram of RBIR and PER}

Figures $6 \mathrm{a}$ and $6 \mathrm{~b}$, using 5000 different channel realizations, show the histogram of RBIR and the frequency of PER as a function of RBIR, respectively, when a MPDU payload of 1500 bytes is transmitted using MCS3 (16-QAM, BCC with $\mathrm{r}=1 / 2$ ) over the TGac B $4 \times 4$ channel with an SNR of $34 \mathrm{~dB}$. The residential TGac B MIMO channel model is a spatial-correlated low-frequency selective with two clusters, maximum excess delay of $80 \mathrm{~ns}$ and root mean square (rms) delay spread of $15 \mathrm{~ns}$ [3, pp. 38]. The RBIR has an average value of 3.39. If the first moment (average) of the RBIR is used in Fig. 3, then a SNR of $13 \mathrm{~dB}$ is obtained. Using this value in Fig. 4, then a negligible PER is estimated (i.e., much smaller than 0.01). However, PHY simulation results outcome a PER of 0.16 when the SNR is equal to 34 $\mathrm{dB}$. This mismatch occurs because in wireless systems operating over MIMO multipath fading channels, the events with low SINR are the ones that generate outage on the system performance and, therefore, use the average RBIR to estimate the PER in MIMO channels is not the appropriate procedure.

On the other hand, Fig. 4 shows that a PER of 0.16 occurs when the SNR is equal to $11.75 \mathrm{~dB}$ and Fig. 3 shows that this value corresponds to a RBIR of 3.2. Finally, using Figures $6 a$ and $6 \mathrm{~b}$, an estimated PER of $0.19(91 / 482)$ is observed when the RBIR is equal to 3.2 , i.e., there is a close agreement with the measured PER of 0.16 obtained with full PHY simulation.

The methodology used to analyze the application of the RBIR technique to abstract the PHY to estimate the MPDU PER in IEEE 802.11ac/ax systems is accomplished by comparing the PER measured using the histograms of RBIR and histograms of PER as a function of RBIR (where both histograms are obtained using complete PHY simulation) with the PER estimated using the PHY abstraction algorithm described in Section III. Notice that our proposed methodology allows to assess the "instantaneous accuracy" of the RBIR ESM technique since the 
estimated PER is based on the aforementioned histograms. Therefore, there is a better agreement with relation to (w.r.t.) the real-world, where a system operating with a target average SNR presents a probabilistically mapping to an ESINR due to the different realizations of the stochastic processes related to the dynamic MIMO channel, noise and interference.

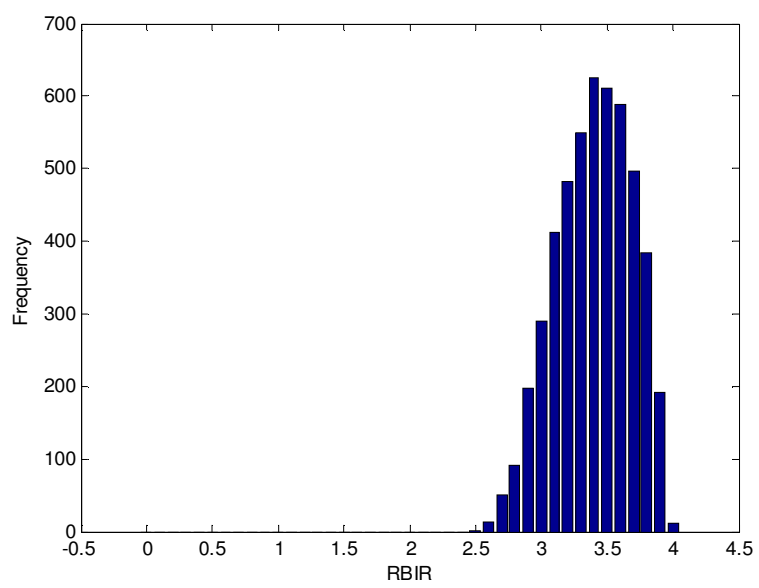

Fig. 6a. Histogram of RBIR for the TGac B 4x4channel with SNR of $34 \mathrm{~dB}$.

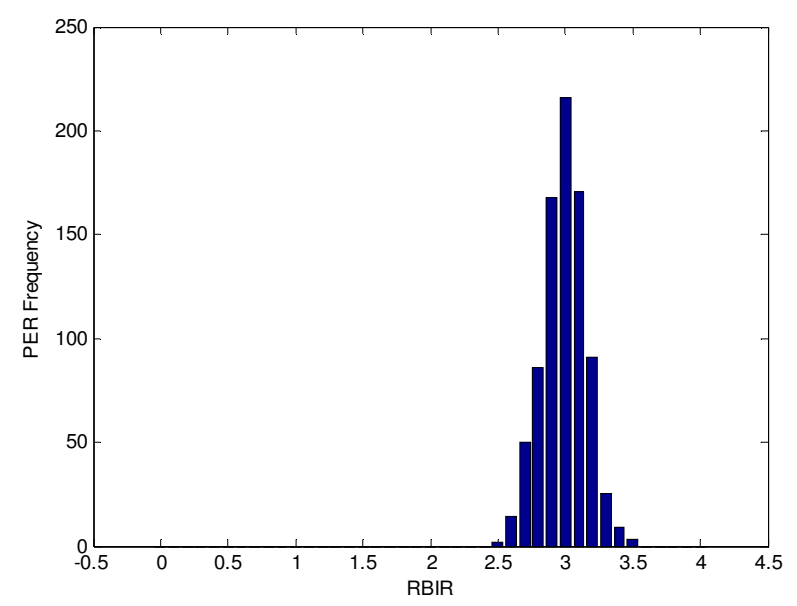

Fig. 6b. Frequency of PER as a function of RBIR.

Finally, observe that our methodology is different from the "long-term accuracy methodology", where a different number of channel realizations as we have done is generated, but for each channel realization the partial statistics of the PER, conditioned for each channel realization, are obtained by different realizations of the noise process (whose variance is set up to obtain a target mean SNR). At the end, the average PER is obtained as the arithmetic mean of the set of PER conditioned to each channel realization. However, this methodology does not necessarily captures the dynamic behavior of real-world timevarying interference system, since there is a high probability of not going through all noise realizations for one channel realization due to the time variant nature of wireless channels.

\section{B. ESINR for PER Estimation: SU-MIMO}

Fig. 7 shows the PER as a function of ESINR, where the TGac B $4 \times 4$ MIMO channel, realistic synchronization and channel estimation schemes, and linear MMSE MIMO receiver are assumed. These results allow to infer that the abstraction of the physical layer using the ESINR mapping based on the RBIR technique can be an effective technique since a difference less than $1 \mathrm{~dB}$ between the PER obtained using the PHY abstraction (i.e., results shown by bold geometric figures) and PER obtained with link level simulation (i.e., results shown by white geometric figures) is observed. Technical reports have shown a mismatching of less than $0.6 \mathrm{~dB}$ when perfect synchronization and channel estimation schemes are assumed over MIMO 2x2 TGac B and D channels [7, 14]. Finally, notice that the use of tuning at Eq. (1) can improve the matching between the simulation results with and without PHY abstraction techniques.

The performance of BCC with soft-decision Viterbi decoding depends on the MPDU packet length. Therefore, an MPDU payload of 1000 bytes is assumed in Fig. 8, instead of a payload of 1500 bytes as assumed in the earlier figures, in order to evaluate the performance of RBIR technique with different packet lengths.

Fig 8 shows on the right side the PER as function of SNR over TGac B 2x2, TGac D 2x2 and TGac D 4x4 MIMO channels, while on the left side the PER as a function of ESINR is depicted. The following setup is assumed: BW of $20 \mathrm{MHz}$; MCS3 (16-QAM, BCC with $\mathrm{r}=1 / 2$ ); LS channel estimation; MMSE receiver, soft-decision Viterbi decoding. The typical office TGac D channel is a spatial-correlated and highly frequency selective channel model with three clusters, maximum excess delay of $390 \mathrm{~ns}$ and rms delay spread of $50 \mathrm{~ns}$ [3, pp. 38]. 


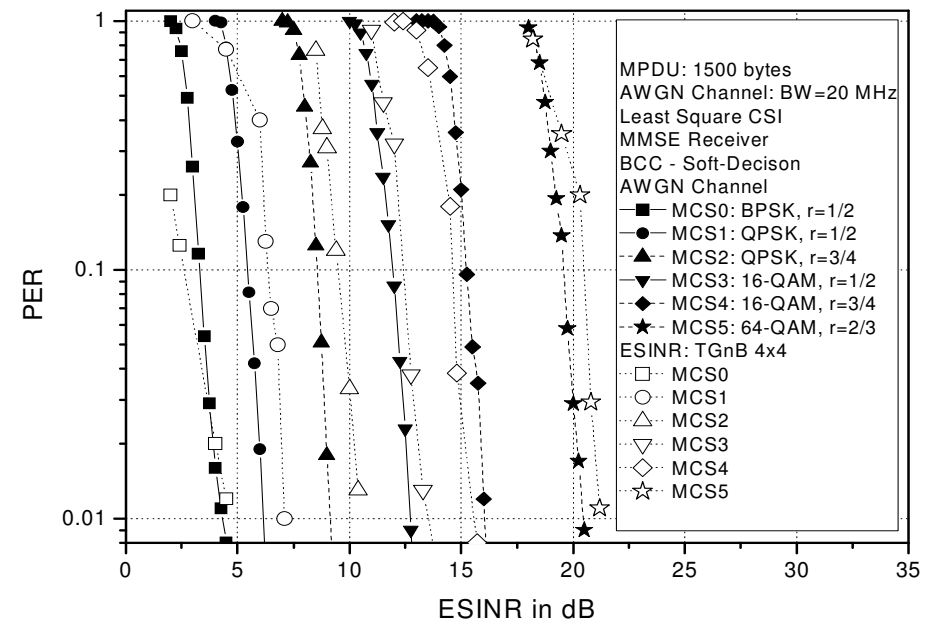

Fig. 7. Comparison between the PER obtained using the equivalent AWGN channel (white geometric figures) and the PER for the TGac B $4 \times 4$ channel using ESINR based on RBIR technique. MPDU payload of 1500 bytes.

Notice that in the full PHY simulation the results are expressed as function of SNR, while is necessary to use the ESINR when the PHY layer abstraction is utilized. Basically, these results ratify, using a different MPDU payload length, that the techniques applied to obtain the ESNR using the RBIR paradigm allow estimating the PER with the adequate precision for system level simulation since there is a mismatching of less than $1 \mathrm{~dB}$ between the estimated ESINR over the MIMO channels and the ESINR used to estimate the PER over the AWGN channel. Observe that an ESINR of $12.5 \mathrm{~dB}$ is demanded to obtain a PER of $1 \%$ over the AWGN channel. For the TGac D 2x2 channel, a SNR of $28 \mathrm{~dB}$ is necessary to obtain this ESINR of $12.5 \mathrm{~dB}$, while an intangible SNR of $44 \mathrm{~dB}$ is necessary when the TGac D $4 \times 4$ is simulated.

It has used in Fig. 8 a TUL to map the ESINR (the $S N R$ is equal to the ESINR for the AWGN channel) to the PER assuming a packet length of 1000 bytes. Notice that the data contained in this TUL is similar to the data shown in Fig. 4, except that this figure refers to a packet length of 1500 bytes. Therefore, ideally we need to have AWGN curves for all possible packet lengths, which is unfeasible. The TGax has proposed to use only two reference curves to interpolate the PER for different packet length, i.e.,

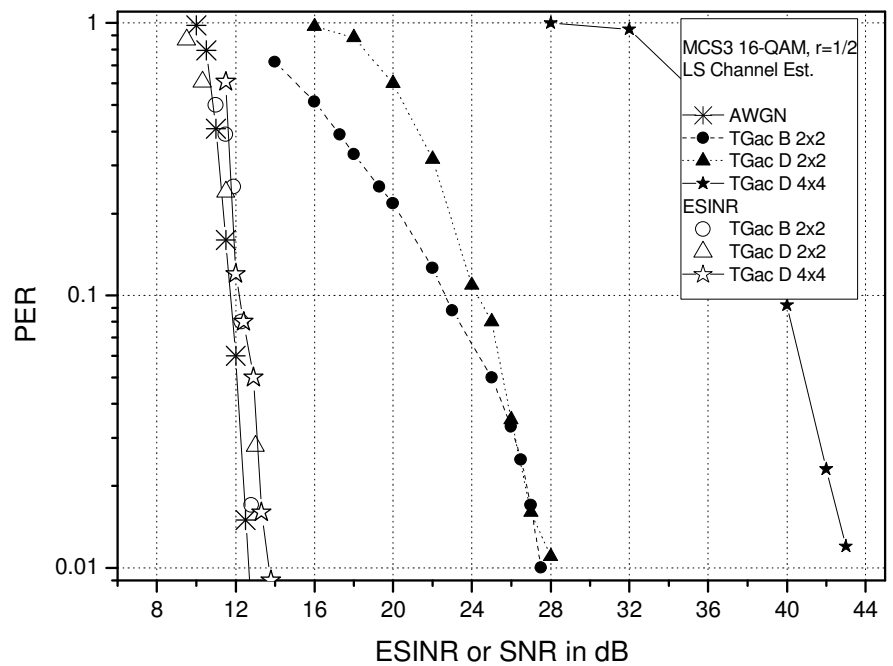

Fig. 8. Comparison between the PER obtained as a function of SNR (right curves) with the PER obtained as function of ESINR (left curves) for MCS3. BW of $20 \mathrm{MHz}$. MPDU payload of 1000 bytes

$$
P E R_{L}=1-\left(1-P E R_{L_{0}}\right)^{\frac{L}{L_{0}}},
$$

where $P E R_{L}$ and $P E R_{L_{0}}$ denote the PER for packet length $L$ and $L_{o}$, respectively [24]. The reference curves that map the ESNIR to $P E R_{L_{0}}$ are generated for two reference packet lengths: 32 bytes for packets less than 400 bytes and 1500 bytes otherwise. The $P E R_{L}$ for different packet lengths is estimated using (6).

Fig. 9 shows results that use a reference packet of 1500 bytes $\left(L_{0}\right)$ to interpolate a PER for a packet length of 1000 bytes $(L)$, where a TGac B 2x2 channel and BW of $20 \mathrm{MHz}$ are assumed. For a PER of $1 \%$, the mismatching between the estimated PER using the RBIR PHY layer abstraction technique (white geometric curves) and the PER estimated using PHY layer simulation (black geometric curves) goes from $2 \mathrm{~dB}$ (MCS1) to close agreement for both MCS0 and MCS3. This figure also shows results for the PER as a function of SNR (bold curves). Notice that commercial $\mathrm{WiFi}$ products present a maximum SNR between $30-35 \mathrm{~dB}$ due to the noise floor of the analog front-end [3, pp. 151]. Finally, we observe that the results shown in [24] indicate a mismatching of approximately $1 d B$ between the PER obtained using the right AWGN reference curve and the PER estimated using (6). However, perfect CSI and synchronization are assumed in [24]. 


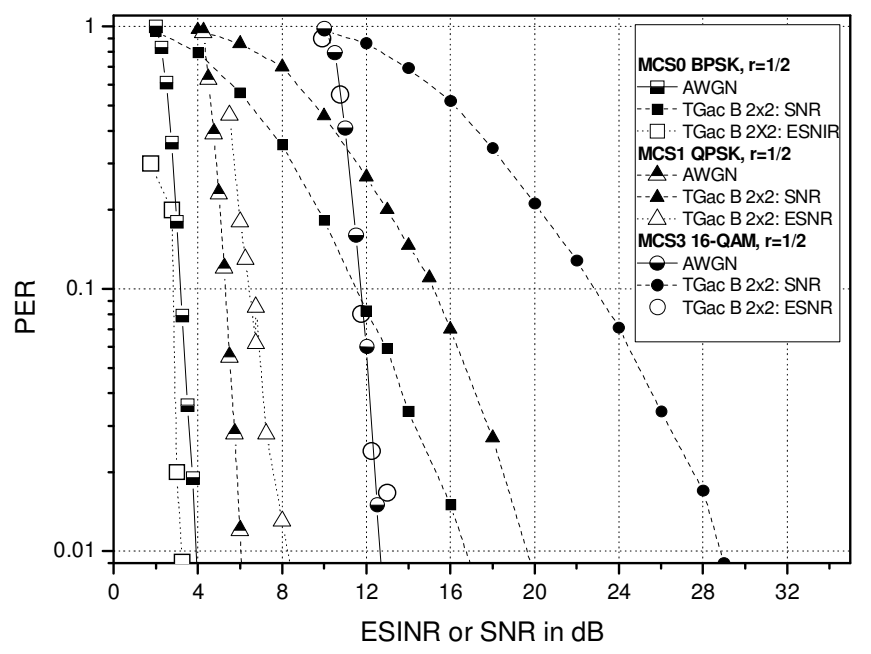

Fig. 9. PER obtained as a function of SNR (right curves) with the PER obtained as function of ESINR (left curves) for MCS0, MCS2 and MCS3 using Eq. (6), with reference packet of 1500 bytes. BW of $20 \mathrm{MHz}$. MPDU payload of 1000 bytes.

\section{ESINR for PER Estimation: $M U-M I M O$}

The description of MU-MIMO channel models, block diagonalization (BD) precoding scheme and MMSE MIMO detector used in this paper can be found in [19].

Fig. 10 shows the PER as a function of SNR and ESINR for IEEE 802.11ac systems operating over the canonical i.i.d. flat fading (Fig. 10a) and TGac D (Fig. 10b) MU-MIMO channels. The following configuration is assumed: [4,2,2,2] (4 transmit antennas, 2 receive antennas per STA, 2 STAs; 2 SSs per STA); BD pre-coding; realistic synchronization and LS channel estimation scheme; linear MMSE MIMO detector; soft-decision Viterbi decoding; BW of $80 \mathrm{MHz}$ and MPDU payload of 1500 bytes. The right side of Figures 10.1 and 10.2 show the PER as function of SNR for MCS0 to MCS3, while on the left side is shown the PER as a function of ESINR. Notice the slope of the curves of PER as a function of SNR (bold geometric figures) is greater (i.e., higher diversity order) for the TGac D channel (Fig. 10b) in relation to the canonical MIMO channel. The positive effects of frequency selectivity of TGac D channels on the performance of BCC explain why these channels present performance gains for a PER of $1 \%$ in relation to the canonical MIMO channel in spite of TGac D channels have spatial correlation on both transmit and receive sides.

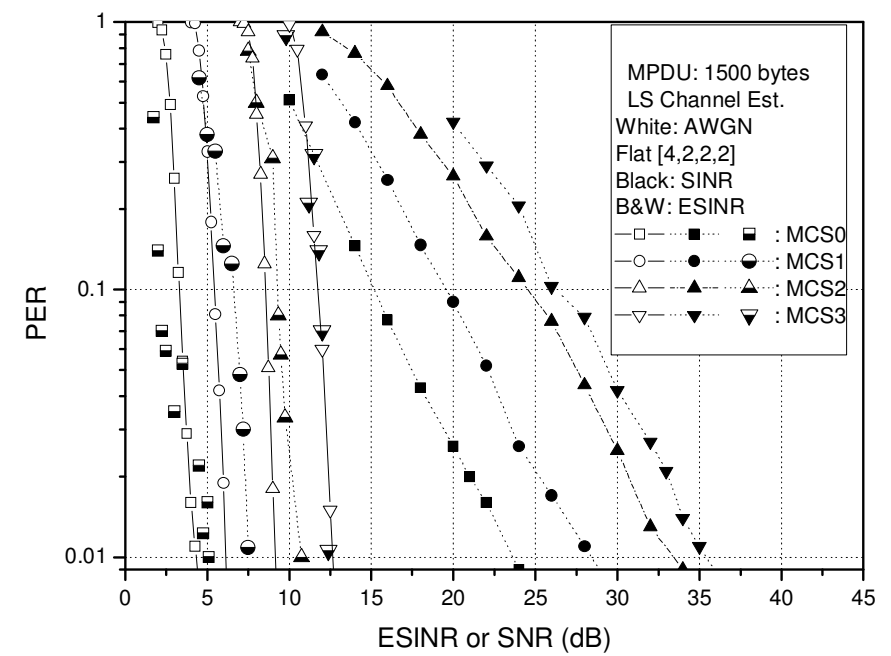

Fig. 10a. Flat Fading $[4,4,2,2]$ MIMO channel.

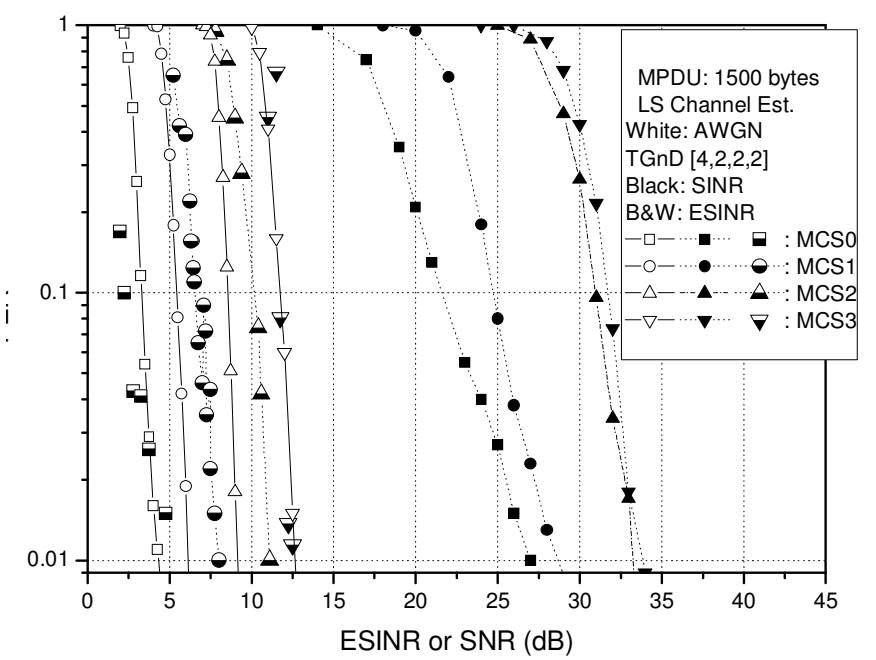

Figure 10b. TGac $[4,4,2,2]$ MIMO channel.

Fig. 10. Comparison between the PER obtained as a function of SNR (right curves) with the PER obtained as function of ESNR (left curves). BW=80 MHz.

The results on the right side of Fig. 10 indicate that the performance of MCS2 (QPSK, BCC with $r=1 / 2$ ) is similar to that one obtained with MCS3 (16-QAM, BCC with $r=2 / 3$ ). However, the ESINR obtained for these MCS is different, as shown on the left side of these figures. This is not an inconsistence since the mapping from RBIR to ESINR depends on the MCS as well as the SINR measured at MMSE MIMO detector output. These results also show a good matching between the PER obtained using PHY simulation and the PER obtained with RBIR abstraction technique for MCS0 and MCS3. However, there is a mismatching of approximately 2 $\mathrm{dB}$ for both MCS1 and MCS2. Therefore, comparing 
these results with those ones shown for SU-MIMO system (according with Figs. from 7 to 9), we have concluded that for MU-MIMO additional procedures are necessary in order to tune Eq. (1) if greater accuracy is demanded. On the other hand, stochastic models for wireless channels are only first-order approximations of the physical world and simulation results are extensively used not to obtain absolute results, but for providing insights on system performance and design options.

\section{LINK LEVEL ADAPTATION USING RBIR ESM}

The objective of link level adaption schemes is to determine in real time the MCS used to transmit the packets based on the CSI. A channel aware adaptive modulation scheme that uses the RBIR ESM is described as follows:

Step 1. Determine the $\operatorname{SINR}_{n, k}$ at the output of MIMO detector for all SSs $\left(n=1, \ldots, N_{s s}\right)$ and SCs $\left(k=1, \ldots, N_{s c}\right)$.

Step 2. Use the steps from 2 to 5 of the algorithm described at item E of Section III to determine the PER for all MCS.

Step 3. Select the MCS which the estimated PER satisfies the target PER (e.g., 1\% PER).

Step 4. Calculate the throughput for the selected MCS as follows:

$$
\mathrm{R}(\mathrm{MCS})=(1-\mathrm{PER}) \cdot \operatorname{rate}(\mathrm{mcs}),
$$

where rate(mcs) is the gross bit rate specified in the IEEE 802.11ac amendment for each MCS. Notice that for each MCS this rate depends upon BW, number of SS and cyclic prefix length.

Step 5. Select the MCS that maximizes (6).

The MCS adaptation in the SU-MIMO case can use the acknowledgment (ACK) control frame (or the compressed feedback frame if the channel sounding is performed) in order to piggyback the information necessary to link adaptation (e.g., MCS, SNR). For MU-MIMO configuration, we have proposed in [24] a protocol where the beamformer, after receiving the CSI feedback from the beemformes, transmit a short frame precoded with the received CSI to assess the link quality. The beamformes then estimate the MCS that optimize the system performance and feed back this information using a short control frame. Based upon over the air experiments, this scheme shows performance improvement in spite of the overhead due to the new sounding and feedback control frames.

Fig. 11 shows the PHY layer throughput as a function of SNR for $2 \times 2$ AWGN as well as TGac $2 \times 2$ and $4 \times 4$ MIMO channels. These results are obtained by performing link level simulations in order to get the range of SNR demanded to obtain a PER of $1 \%$, assuming a BW of $80 \mathrm{MHz}$ and a MPDU payload of 1500 bytes. The values of the gross PHY layer throughput for 2 and 4 SSs are obtained using the tables shown in [1,3]. Notice that the SNR and ESINR have the same logical meaning for MIMO AWGN channels and the procedure described in Section III can be used for mapping SNR to ESINR for TGac B MIMO channels.

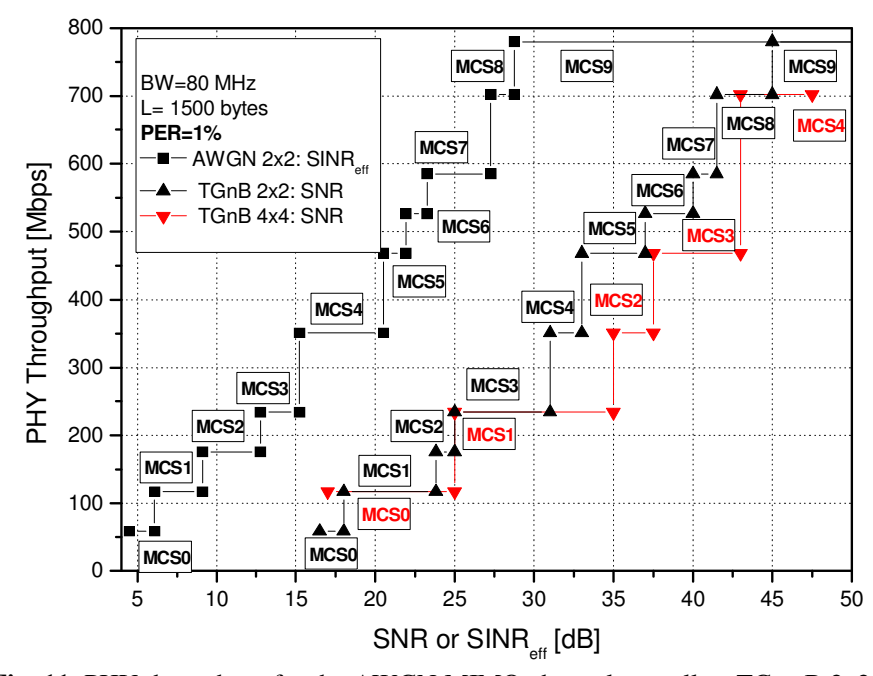

Fig. 11. PHY throughput for the AWGN MIMO channel as well as TGac B 2x2 and $4 \times 4$ channels as a function of SNR for a PER of $1 \%$.

The curve with bold triangles on the right side of Fig. 11 shows the MCS that maximize the PHY throughput as function of SNR for the SU-MIMO TGac B 2x2 channel. The results on the left side show the throughput as a function of ESINR over the MIMO 2x2 AWGN channel. The curve with inverted triangles show on right side of Fig. 11 is similar to the curve with bold triangles, except that the SU-MIMO TGac B $4 \times 4$ channel is assumed. Observe that the PHY throughput of $351 \mathrm{Mbps}$ is obtained using either MCS4 with 2 SS or MCS2 with 4 SS over the TGac B $4 \times 4$ channel. Finally, notice that the link level adaptation scheme must first estimate in real-time the effective SINR for each channel realization and then use the ESINR to select the MCS that maximizes the 
throughput according with the degrees of freedom of each channel configuration.

\section{CONCLUSIONS}

First, we described the motivations that drive R\&D on the application of PHY abstraction techniques for system level simulation in the framework of IEEE 802.11ax specification. Second, we developed in great detail the application of the received bit information rate (RBIR) technique used to calculate the ESINR as well as the mapping from ESINR to PER. Third, we showed that our simulation results present a good agreement with those ones publicized by the TG $802.11 \mathrm{ax}$, where perfect synchronization and channel estimation are assumed. Next, we verified that implementing realistic channel estimation and synchronization schemes cause performance losses that range from 3 to $3.5 \mathrm{~dB}$ due to imperfect synchronization and CSI even over MIMO AWGN channels. Simulation results have shown that the application of PHY layer abstraction techniques based on RIBR can be adequate to provide first order insights on IEEE 802.11ac/ax system level performance and design options with low computational burden for both SU and MU-MIMO channels with imperfect synchronization and CSI. Finally, we proposed a channel aware adaptive MCS scheduling scheme based on the RBIR metric. The PHY layer abstraction technique investigated in this paper has also been used to reduce the computational burden when over the air experiments are used to design MU beamforming protocols that increase the resilience against downlink interference due to imperfect channel estimation [25].

\section{REFERENCES}

[1] Wireless LAN Medium Access Control and Physical Layer Specifications, Amendment 5: Enhancement for Very High Throughput for Operations in Bands below 6 GHz. IEEE P802.1 lac, Dec. 2013.

[2] Wireless LAN Medium Access Control and Physical Layer Specifications, Amendment 4: Enhancement for Higher Throughput. IEEE Std 802.11n-2009, June 2009.

[3] E. Perahia and R. Stacey, Next Generation Wireless LANS: 802.11n and 802.11ac.2th ed. Cambridge: Cambridge University Press, 2013.

[4] O. Bejarano, E. W. Knightly and M. Park. "IEEE 802.11ac: from channelization to multi-user MIMO," IEEE Communications Magazine, vol. 51, no. 10, pp. 84-90, Oct. 2013, doi: 10.1109/MCOM.2013.6619570.

[5] F. M. Abinader et. al. "Enabling the coexistance of LTE and Wi-Fi in unlicensed bands", in IEEE Communications Magazine, vol. 52, no. 11, pp. 54-61, Nov. 2014, doi: 10.1109/MCOM.2014.6957143.

[6] D-J. Deng, K-C. Cheng and R-S. Cheng. "IEEE 802.11ax: next generation wireless local area networks," in 10th 2014 International Conference on Heterogeneous Networking for Quality, Reliability, Security and Robustness, 2014, doi: 10.1109/QSHINE.2014.6928663

[7] IEEE 802.11-14/0527-r1, "PHY Layer Abstractions for TGax System Level Simulations," May 2014.

[8] 3GPP TSG-RAN-1 Meeting \#35, R1-03-1298, "Effective SIR Computation for OFDM System-Level Simulations", Nov. 2003.

[9] 3GPP2-C30-20030429-010, "Effective-SNR Mapping for Modeling Frame Error Rates in Multiple-State Channels", Nov. 2003.

[10] IEEE 802.16m-08/004r5, "IEEE 802.16m Evaluation Methodology Document",2009.

[11] R. Santos, W. Freitas , E. Stancanelli and F. Cavalcanti, "Link-tosystem level interface solutions in multistate channels for 3GPP LTE wireless system," in XXV Brazilian Symposium of Telecommunications, 2007.

[12] J. C. Ikuno, M. Wrulich, M. Rupp, "System level simulation of LTE networks," in IEEE 71th Vehicular Technology Conference, Ottawa, 2010, doi: 10.1109/VETECS.2010.5494007.

[13] IEEE 802.11-04/0174r1, "PHY Abstraction for System Simulation," Feb. 2004

[14] IEEE 802.11-13/0117r0, "PHY Abstraction for HEW System Level Simulation", Jan. 2014.

[15] L. Wan, S. Tsai and M. Almgren, "A fading-insensitive metric for unified link quality model," in IEEE Wireless Network Conference 2006 (WCNC 2006), April 2006, doi: 10.1109/WCNC.2006.1696622.

[16] IEEE 802.11-14/0571r9, "IEEE P802.11 Wireless LANs: 11ax Evaluation Methodology, " May 2015.

[17] J. Wu, Z. Yin, J. Zhang and W. Heng, "Physical layer abstraction algorithms research for 802.11n and LTE downlink," in International Symposium on Signals, Systems and Electronics (ISSSE 2010), Nanjing, China, 2010, doi: 10.1109/ISSSE.2010.5607125.

[18] D. Nojima, L. Lanante Jr., Y. Nagao, M. Kurosaki and H. Ochi, "Performance evaluation for multi-user MIMO IEEE 802.11 ac wireless LAN system," in 14 International Conference on Advanced Communication Tecnhology, Feb. 2012.

[19] R. P. F. Hoefel. "Multi-User OFDM MIMO in IEEE 802.11ac WLAN: a simulation framework to analysis and synthesis", in IEEE Latin America Transactions, vol.13, no. 2, pp. 540-545, Feb. 2015, doi: 10.1109/LatinCom.2013.6759823.

[20] R. P. F. Hoefel, "IEEE 802.11n: On the performance of channel estimation schemes over OFDM MIMO spatially-correlated frequency selective fading TGn channels," in XXX Brazilian Symposium on Telecommunications, Brasília, Brazil, 2012.

[21] IEEE 802.11-13/1131r0, "PHY Abstraction for HEW System Level Simulation", Sept. 2013.

[22] IEEE 802.11-14/0330r2, "PHY Abstraction," March 2014.

[23] G. Breit, H. Sampath, S. Vermani, et. al., TGac Channel Model Addendum Support Material. IEEE 802.11-09/06/0569r0, May 2009.

[24] IEEE 802.11-14/0647r2, "PHY Abstraction Method Comparison," May, 2014.

[25] O. Bejarano, R. P F. Hoefel, E. Knightly. "Resilient, multi-user beamforming WLANs: mobility, interference and imperfect CSI," in IEEE International Conference on Computer Communications (IEEE INFOCOM 2016), San Francisco, USA, 2016, doi: 10.1109/INFOCOM.2016.7524573

[26] R. P F. Hoefel, O. Bejarano. "On Application of PHY Layer Abstraction Techniques in IEEE 802.11ac/ax WLANs," in XXXIII Simpósio Brasileiro de Telecomunicações (SBrT'15), Juiz de Fora, MG, Brazil, September 1-4, 2015 
Roger Pierre Fabris Hoefel is an Associate Professor at Department of Electrical Engineering of Federal University of Rio Grande do Sul (UFRGS), Porto Alegre, Rio Grande do Sul, Brazil. He received the degree of Doctor in Electrical Engineering by State University of Campinas (UNICAMP), Campinas, São Paulo, Brazil, in 2000; Master in Computer Science by UFRGS in 1994; Post-Graduated in Electronic Instrumentation by Federal University of Santa Catarina (UFSC), Florianópolis, Santa Catarina, Brazil, in 1991; Bachelor in Electrical Engineering by Pontific Catholic University of Rio Grande do Sul (PUC-RS), Porto Alegre, Rio Grande do Sul, Brazil, in 1990. His current research interests include design, analyzes and optimization of algorithms for physical and medium access control layers of wireless networks.

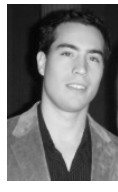

Oscar Bejarano received his Ph.D. and M.S. from Rice University and his B.S. from the University of Texas at Austin; all degrees are in Electrical and Computer Engineering. Since 2015, he has been a wireless systems engineer at Cisco Systems. His current research interests include cross-layer protocol design for multi-antenna systems, as well as RF localization. 